\title{
FACTORIZATION FOR BOUNDED ANALYTIC FUNCTIONS IN THE UNIT DISK AND AN APPLICATION TO PRIME IDEALS
}

\author{
RAYMOND MORTINI \\ (This paper is dedicated to the memory of Professor Gerald Schmieder)
}

\begin{abstract}
We prove several factorization theorems for bounded analytic functions in the open unit disk and present a very simple new proof of two conjectures of Frank Forelli and the author on the structure of finitely generated, respectively countably generated prime ideals in $H^{\infty}$.
\end{abstract}

\section{Introduction}

Let $T=\{z \in \mathrm{C}:|z|=1\}$ be the unit circle. For $f \in H^{\infty}$, the space of all bounded analytic functions in the open unit disk $D=\{z \in C:|z|<$ $1\}$, let $E(f)=\left\{\alpha \in T: \lim _{r \rightarrow 1} f(r \alpha)=0\right\}$. It easily follows from an extension of Hoffman's factorization theorem due to Izuchi ([6], p. 55) that any Blaschke product $B$ can be written as $B=C D$, where $B$ and $C$ are two Blaschke products such that $E(B)=E(C)=E(D)$. This type of factorization theorem plays a fundamental role in the description of the ideal structure of $H^{\infty}$. Now suppose that $f$ is a bounded analytic function in the unit disk with $\|f\|:=\sup _{|z|<1}|f(z)| \leq 1$ such that $\lim _{r \rightarrow 1} f(r)=0$. Does there exist, within $H^{\infty}$, two factors $g$ and $h$ of $f$ such that $f=g h$ and $\lim \sup _{r \rightarrow 1}|h(r)|=$ $\lim \sup _{r \rightarrow 1}|g(r)|=1$ ? The example of the atomic inner function $S(z)=$ $\exp \left(-\frac{1+z}{1-z}\right)$ shows that, in general, the answer is no. In fact, the classical RieszSmirnov factorization theorem [2] tells us that (inside the algebra $H^{\infty}$ ), the only factors of $S$ are the roots $S^{\alpha}$ of $S(0 \leq \alpha \leq 1)$ (modulo unimodular constants, of course); but those all tend to 0 as $r \rightarrow 1$. What about, e.g., the Blaschke product $B$ with zeros $r_{n}=1-n^{-2}$ ? It is easily seen that $\lim _{r \rightarrow 1} B(r)=0$. A result of Øyma [12] shows that every Blaschke product indeed, admits a factorization of the desired type. For a variant of this, see also [4]. What about general singular inner functions or outer functions? 
Let $f$ be a function in the unit ball $\mathscr{B}=\left\{f \in H^{\infty}:\|f\| \leq 1\right\}$ of $H^{\infty}$. A sequence $\left(z_{n}\right)$ in D with $\left|z_{n}\right| \rightarrow 1$ is called a spectral sequence for $f$ if $\lim _{n} f\left(z_{n}\right)=0$. Let us say that the function $f \in \mathscr{B}$ is $\left(z_{n}\right)$-factorizable, if there exists $g, h \in \mathscr{B}$ such that $f=g h$ and $\lim \sup \left|h\left(z_{n}\right)\right|=\lim \sup \left|g\left(z_{n}\right)\right|=1$ whenever $\left(z_{n}\right)$ is a spectral sequence. It is the aim of this note to give a simple characterization of all factorizable functions in $H^{\infty}$. Besides the known case of Blaschke products [4], it will be shown that outer functions as well as singular inner functions with associated continuous measure are factorizable. Concerning discrete singular inner functions, the result is more delicate and depends on the sequence $\left(z_{n}\right)$ itself. As an application we present a very simple new proof of two conjectures of F. Forelli and the author on finitely resp. countably generated prime ideals in $H^{\infty}$. The known proofs [8], [9], [3] for the finitely generated case and [4] for the countably generated case all use Hoffman's theory on the structure of the maximal ideal space of $H^{\infty}$ and, in the second case, Suarez's result that $H^{\infty}$ is a separating algebra. Here we need only the assertion of the corona theorem that the unit disk is dense in the spectrum of $H^{\infty}$.

\section{Factorizable functions}

Let $f \in \mathscr{B}$, the unit ball of $H^{\infty}$. According to the canonical factorization theorem for functions in Hardy spaces, either $f \equiv 0$ or $f$ can be written as $f=e^{i \theta} B F_{\mu}$, where $\theta \in[0,2 \pi[, B$ is a Blaschke product and where the zero-free function $F_{\mu}$ is given by

$$
F_{\mu}(z)=\exp \left(\int_{|\xi|=1} \frac{z+\xi}{z-\xi} d \mu(\xi)\right) .
$$

Here $\mu=\mu_{s}+\log \left(\frac{1}{\mid f(\xi)}\right) d \sigma$ for some positive, finite Borel measure $\mu_{s}$ singular to normalized Lebesgue measure $\sigma$ on $T$. The support of $\mu$ is the smallest compact subset of $T$ for which $\mu(K)=\mu(T)$. Note that if $f$ is not inner, then the support of $\mu$ is $T$. We first give a sufficient condition for factorizability.

Theorem 1.1. Let $f=B F_{\mu} \in \mathscr{B}, f \not \equiv 0$. Suppose that $\left(z_{n}\right)$ is a sequence in $\mathrm{D}$ that converges to a point $\alpha \in T$ with $\mu_{s}(\{\alpha\})=0$. Then there exists $g$ and $h \in \mathscr{B}$ with $f=g h$ and $\lim \sup \left|h\left(z_{n}\right)\right|=\lim \sup \left|g\left(z_{n}\right)\right|=1$.

Proof. To simplify notation, let $\alpha=1$. Choose a nested sequence of closed $\operatorname{arcs} J_{n}$ centered at 1 with $J_{0}=T$ and $\left|J_{n}\right| \rightarrow 0$, where $|J|$ denotes arclength of the $\operatorname{arc} J$. Let $I_{n}=J_{n} \backslash J_{n+1}$. Note that $I_{n}$ is a union of two arcs. Define the restriction measure $\mu_{n}:=\left.\mu\right|_{I_{n}}$. Since $\mu(\{1\})=0$, we have $\sum_{n=0}^{\infty} \mu_{n}=\mu$. It 
is easy to see that due to the relation $\left|1-e^{z}\right| \leq|z|$ for $z \in \mathrm{C}$ with $\operatorname{Re} z \leq 0$, the functions

$$
G_{n}(z)=\exp \left(\int_{J_{n}} \frac{z+\xi}{z-\xi} d \mu(\xi)\right)
$$

satisfy

$$
\left|1-G_{n}(z)\right| \leq \frac{1+|z|}{1-|z|} \mu\left(J_{n}\right),
$$

and that outside the support of $\mu, F_{\mu}$ is unimodular on $T$. The proof now proceeds along the same line as that for Blaschke products (see [4]). Choose two distinct subsequences $\left(s_{n}\right)$ and $\left(r_{n}\right)$ of $\left(z_{n}\right)$ so that $s_{n} \rightarrow \alpha=1$ and $r_{n} \rightarrow \alpha=1$. Let $\left(a_{n}\right)$ denote the zero sequence of $B$. Write $L_{n}(z)=\frac{\left|a_{n}\right|}{a_{n}} \frac{a_{n}-z}{1-\bar{a}_{n} z}$ and

$$
F_{n}(z)=\exp \left(\int_{I_{n}} \frac{z+\xi}{z-\xi} d \mu(\xi)\right) .
$$

Finally, let $f_{n}=L_{n} F_{n}$. Then $B F_{\mu}=\prod_{n=0}^{\infty} f_{n}$. Note that by (1.1) the convergence is absolute, hence unconditional, and local uniform in $D$. Moreover, $\left\|f_{n}\right\| \leq 1$ and $\prod_{j \geq n} F_{j}=G_{n}$.

We shall inductively construct certain subsequences $\left(S_{n}\right)$ of $\left(s_{n}\right)$ and $\left(R_{n}\right)$ of $\left(r_{n}\right)$, associated with sequences of integers $\left(m_{j}\right)$ and $\left(n_{j}\right)$ satisfying $m_{j}<$ $n_{j}<m_{j+1}$ so that the functions

$$
g=\prod_{j=1}^{\infty}\left(\prod_{k: m_{j} \leq k<n_{j}} f_{k}\right)
$$

and

$$
h=\prod_{j=1}^{\infty}\left(\prod_{k: n_{j} \leq k<m_{j+1}} f_{k}\right)
$$

satisfy $\lim \left|g\left(S_{j}\right)\right|=\lim \left|h\left(R_{j}\right)\right|=1$.

Start with $m_{1}=0$ and let $\left(\eta_{n}\right)$ be an increasing sequence of positive numbers with $\lim \eta_{n}=1$. Suppose that $m_{j}$ is given. Choose $R_{j} \in\left\{r_{k}: k \in \mathrm{N}\right\}$ so that

$$
\left|\prod_{k \leq m_{j}} f_{k}\left(R_{j}\right)\right|>\eta_{j}
$$

This can be done since the functions $\prod_{j=0}^{N} F_{j}$ and the finite Blaschke products $\prod_{j=0}^{N} L_{j}$ are unimodular and analytic on a small arc around the cluster point 1 of the $r_{n}$. 
Then choose $n_{j}>m_{j}$ so that

$$
\left|\prod_{k \geq n_{j}} f_{k}\left(R_{j}\right)\right|>\eta_{j}
$$

This can be done since the tails of infinite products converge locally uniformly to 1 . Let $S_{j} \in\left\{s_{k}: k \in \mathrm{N}\right\}$ be chosen so that

$$
\left|\prod_{k \leq n_{j}} f_{k}\left(S_{j}\right)\right|>\eta_{j}
$$

Finally choose $m_{j+1}>n_{j}$ so that

$$
\left|\prod_{k \geq m_{j+1}} f_{k}\left(S_{j}\right)\right|>\eta_{j}
$$

Then the functions $g$ and $h$ as defined in (1.2) and (1.3) do the job. Indeed, for fixed $j$, we have

$$
\left|g\left(S_{j}\right)\right| \geq\left|\prod_{v: v<n_{j}} f_{v}\left(S_{j}\right)\right|\left|\prod_{v: v \geq m_{j+1}} f_{v}\left(S_{j}\right)\right| \geq \eta_{j}^{2}
$$

and

$$
\left|h\left(R_{j}\right)\right| \geq\left|\prod_{v: v<m_{j}} f_{v}\left(R_{j}\right)\right|\left|\prod_{v: v \geq n_{j}} f_{v}\left(R_{j}\right)\right| \geq \eta_{j}^{2}
$$

Hence $\lim \sup \left|g\left(z_{n}\right)\right|=\lim \sup \left|h\left(z_{n}\right)\right|=1$. Since $f=g h$, we are done.

As an immediate consequence, we have

COROLlary 1.2. a) Let $f$ be an outer function of norm one, or a singular inner function associated to a continuous measure, or a Blaschke product satisfying $\lim _{r \rightarrow 1} f\left(r e^{i \theta}\right)=0$ for some $\theta \in[0,2 \pi[$. Then $f$ admits a factorization $f=g h$, where

$$
\limsup _{r \rightarrow 1}\left|g\left(r e^{i \theta}\right)\right|=\limsup _{r \rightarrow 1}\left|h\left(r e^{i \theta}\right)\right|=1 .
$$

b) The same holds if $f=S_{v}$ is a discrete singular inner function for which $e^{i \theta}$ is a point of the support of $v$ with $v\left(\left\{e^{i \theta}\right\}\right)=0$.

We are now able to characterize the functions that are not $\left(z_{n}\right)$-factorizable. 
Theorem 1.3. Let $f=B F_{\mu} \in \mathscr{B}, f \not \equiv 0$. Suppose that $\left(z_{n}\right)$ is a spectral sequence for $f$, or more generally, any sequence in $\mathrm{D}$ converging to the boundary of $\mathrm{D}$. Then $f$ is not $\left(z_{n}\right)$-factorizable if and only if $\left(z_{n}\right)$ converges to a point $\alpha \in T$ with

$$
\mu_{s}(\{\alpha\})>0 \quad \text { and } \quad \liminf \frac{1-\left|z_{n}\right|^{2}}{\left|\alpha-z_{n}\right|^{2}}>0 .
$$

PRoOF. Suppose that $\lim z_{n}=\alpha$ and that $\left(z_{n}\right)$ satisfies (1.4). Hence $\frac{1-\left|z_{n}\right|^{2}}{\left|\alpha-z_{n}\right|^{2}}$ $\geq \varepsilon>0$ for all $n$. Let $f=g h$ be any factorization of $f$ with $g, h \in \mathscr{B}$. Since $\mu_{s}(\{\alpha\})>0$, at least one of the functions $g$ or $h$, say $g$, must have a singular inner factor of the form $S^{\omega}(\bar{\alpha} z)$ for some $\omega>0$, where $S(z)=\exp \left(-\frac{1+z}{1-z}\right)$. But then

$$
\left|g\left(z_{n}\right)\right| \leq\left|S^{\omega}\left(\bar{\alpha} z_{n}\right)\right|=\exp \left[-\omega \frac{1-\left|z_{n}\right|^{2}}{\left|\alpha-z_{n}\right|^{2}}\right] \leq \exp (-\omega \varepsilon) \leq r<1 .
$$

Thus $f$ is not $\left(z_{n}\right)$-factorizable.

To prove the converse, let $f \in \mathscr{B}, f \not \equiv 0$, be a function that is not $\left(z_{n}\right)$ factorizable. Theorem 1.1 shows that the sequence $\left(z_{n}\right)$ cannot cluster at a point $\alpha$ for which $\mu_{s}(\{\alpha\})=0$. Hence we may assume that $\mu_{s}$ has positive mass at every cluster point $\alpha$ of $\left(z_{n}\right)$. We shall show that the non-factorizability implies that $\left(z_{n}\right)$ has only a single cluster point, hence converges to some $\alpha \in T$, and that at this point, $\lim \inf \frac{1-\left|z_{n}\right|^{2}}{\left|\alpha-z_{n}\right|^{2}}>0$. To do this, let $\left(\eta_{n}\right)$ be a subsequence of $\left(z_{n}\right)$ converging to some $\alpha \in T$ and suppose that $\left(\xi_{n}\right)$ is another subsequence of $\left(z_{n}\right)$ that converges to a point $\beta \in T, \beta \neq \alpha$. Obviously $f$ can be written as $f=g h$, where $g$ and $h$ are unimodular and analytic on arcs containing $\alpha$, respectively $\beta$. (One may take, e.g., as $g$ the function $g=B_{1} F_{\mu_{1}}$, where $B_{1}$ is a Blaschke subproduct of $B$ whose zeros do not cluster at $\alpha$ and where $\mu_{1}$ is the measure $\mu$ restricted to an arc centered at $\beta$ but not containing $\alpha$ ). Then $f=g h$ is a factorization with $\lim \sup \left|g\left(z_{n}\right)\right|=\lim \sup \left|h\left(z_{n}\right)\right|=1$. A contradiction. Hence $\left(z_{n}\right)$ has only a single cluster point, say $\alpha$. Again, since $\mu_{s}(\{\alpha\})>0, f(z)=S^{\omega}(\bar{\alpha} z) R(z)$ for some $\omega>0$ and a function $R \in \mathscr{B}$ that has no singular inner factor with positive point mass at $\alpha$. If we assume that $\lim \inf \frac{1-\left|z_{n}\right|^{2}}{\left|\alpha-z_{n}\right|^{2}}=0$, say $\lim \frac{1-\left|z_{n_{n}}\right|^{2}}{\left|\alpha-z_{n_{k}}\right|^{2}}=0$ for some subsequence $\left(z_{n_{k}}\right)$ of $\left(z_{n}\right)$, then $\left|S^{\omega}\left(\bar{\alpha} z_{n_{k}}\right)\right| \rightarrow 1$. Hence, by Theorem 1.1, we obtain the factorization $f(z)=\left(S^{\omega}(\bar{\alpha} z) R^{\prime}(z)\right) R^{\prime \prime}(z)$ with $\lim \sup \left|S^{\omega}\left(\bar{\alpha} z_{n_{k}}\right)\right|\left|R^{\prime}\left(z_{n_{k}}\right)\right|=$ $\lim \sup \left|R^{\prime \prime}\left(z_{n_{k}}\right)\right|=1$, a contradiction. Thus $\liminf \frac{1-\left|z_{n}\right|^{2}}{\left|\alpha-z_{n}\right|^{2}}>0$ and the proof is complete. 


\section{Prime ideals}

The following Theorem has recently [4] been proven using Hoffman's theory on the structure of the maximal ideal space of $H^{\infty}$. Here I reprove it using only the elementary factorization theorem Theorem 1.1 above, together with the simplest version of the Corona Theorem. Recall that $S(z)=\exp \left(-\frac{1+z}{1-z}\right)$ and that $S_{\sigma}(z)=S(\bar{\sigma} z)$. For $f \in H^{\infty}$, we let $\hat{f}$ denote the Gelfand transform of $f$, defined on the maximal ideal space $M\left(H^{\infty}\right)$ of $H^{\infty}$.

THEOREM 2.1. A nonzero prime ideal I in $H^{\infty}$ is countably generated if and only if

$$
I=M\left(z_{0}\right):=\left\{f \in H^{\infty}: f\left(z_{0}\right)=0\right\} \quad \text { for some } z_{0} \in \mathrm{D}
$$

or

$$
I=I\left(S_{\sigma}, S_{\sigma}^{1 / 2}, S_{\sigma}^{1 / 3}, \ldots\right) \quad \text { for some } \sigma \text { with }|\sigma|=1
$$

Proof. Suppose that $I$ is generated by the functions $f_{1}, f_{2}, \ldots$ We may assume, without loss of generality, that $\left\|f_{n}\right\| \leq 2^{-n}$. As usual, if the zero set

$$
Z(I)=\left\{m \in M\left(H^{\infty}\right): \widehat{f}(m)=0 \text { for every } f \in I\right\}
$$

of the ideal meets the unit disk, then $I$ coincides with the principal ideal $z-z_{0}$ for some $z_{0} \in \mathrm{D}$. Hence $I=M\left(z_{0}\right)$. So it remains to study the case where the functions $f_{n}$ have no common zero in D. For $m \in M\left(H^{\infty}\right)$, let $q(m)=\sum_{n=1}^{\infty}\left|\widehat{f_{n}}(m)\right|$. By Gelfand-theory, $q$ is a continuous function. Since $I$ is assumed to be proper, there exists a maximal ideal, hence some $m \in M\left(H^{\infty}\right)$, such that $\widehat{f}_{n}(m)=0$ for every $n$. In particular, $q(m)=0$. The Corona-Theorem now implies that there exists a sequence $\left(z_{k}\right)$ in the unit disk for which $q\left(z_{k}\right) \rightarrow 0$ (otherwise $q$ would be bounded away from zero in $\mathrm{D}$, hence on $M\left(H^{\infty}\right)$ ). By taking a subsequence, we may assume that $z_{k} \rightarrow 1$. In particular, for every $f \in I$, we have $f\left(z_{k}\right) \rightarrow 0$; (note that $f \in I$ implies that $|\widehat{f}| \leq C|q|$ on $M\left(H^{\infty}\right)$ for some constant $C>0$ depending on $\left.f\right)$. If we suppose that the singular inner factor of $f, f \not \equiv 0$, has no atom (at $z=1$ ), then, by Theorem 1.1, $f=g h$ where $\lim \sup \left|g\left(z_{k}\right)\right| \cdot \lim \sup \left|h\left(z_{k}\right)\right|>0$. Hence neither $g$ nor $h$ belongs to $I$. This is a contradiction to the primeness of $I$. Thus $f_{j}=S^{\sigma_{j}} F_{j}$, where $\sigma_{j}>0$ and where the inner factor of $F_{j}$ does not have any atom at $z=1$. Since $I$ is prime and $F_{j} \notin I$, we obtain that $S^{\sigma_{j}} \in I$. Hence, due to primeness again, we see that $I$ is generated by the functions $S, S^{1 / 2}, S^{1 / 3}, \ldots$

The proof above also yields the following result, as has been communicated to the author by T. W. Gamelin: 
THEOREM 2.2. The only nonzero prime ideals in $H^{\infty}$ whose hull (=zero set) are $G_{\delta}$-sets are the ideals

$$
P=M\left(z_{0}\right):=\left\{f \in H^{\infty}: f\left(z_{0}\right)=0\right\} \quad \text { for some } z_{0} \in \mathrm{D}
$$

or

$$
P=I\left(S_{\sigma}, S_{\sigma}^{1 / 2}, S_{\sigma}^{1 / 3}, \ldots\right) \quad \text { for some } \sigma \text { with }|\sigma|=1
$$

\section{REFERENCES}

1. Forelli, F., Divisibility problems in $A$ (D) and $H^{\infty}$ (D), Lecture Notes in Math. 1043 (1984), 397-398. Linear and Complex Analysis Problem Book, 199 research Problems. Ed: Havin, Hrushchev, Nikolski. Berlin, Heidelberg, New York: Springer 1984.

2. Garnett, J., Bounded Analytic Functions, Academic Press, New York 1981.

3. Gorkin, P., Prime ideals in closed subalgebras of $L^{\infty}$, Michigan Math. J. 33 (1986), 315-323.

4. Gorkin, P., Mortini, R., Countably generated prime ideals in $H^{\infty}$, Math. Z. 251 (2005), 523-533.

5. Hoffman, K., Bounded analytic functions and Gleason parts, Ann. of Math. 86 (1967), 74 111.

6. Izuchi, K., Factorization of Blaschke products, Michigan Math. J. 40 (1993), 53-75.

7. Kelleher, J., Review No 1075, MR 55 (1978), 146-147.

8. Mortini, R., Zur Idealstruktur der Disk-Algebra A(D) und der Algebra $H^{\infty}$, Dissertation, Universität Karlsruhe, 1984.

9. Mortini, R., Finitely generated prime ideals in $H^{\infty}$ and A(D), Math. Z. 191 (1986), 297-302.

10. Mortini, R., Gleason parts and prime ideals in $H^{\infty}$, Lecture Notes in Math. 1573 (1994), 136-138. Linear and Complex Analysis Problem Book 3, Ed.: V. Havin, N. Nikolski. Berlin, Heidelberg, New York: Springer 1994.

11. Suarez, F. D., Čech cohomology and covering dimension for the $H^{\infty}$ maximal ideal space, J. Funct. Anal. 123 (1994), 233-263.

12. Øyma, K., Approximation by interpolating Blaschke products, Math. Scand. 79 (1996), 255262.

DÉPARTEMENT DE MATHÉMATIQUES

UNIVERSITÉ PAUL VERLAINE DE METZ

ILE DU SAULCY

F-57045 METZ

FRANCE

E-mail: mortini@univ-metz.fr 